\title{
Alteraciones del sueño asociadas a patología mental en pacientes con osteoartritis de rodilla o de cadera
}

\author{
Heimo Viinamäki, Profesor* \\ Peca Mäntyselkä, Investigador** \\ Esko Kumpusalo, Senior lecturer** \\ Jussi Kauhanen, Lecturer** \\ Pirjo Halonen, Investigador ${ }^{\star \star *}$ \\ Jorma Takala, Profesor** \\ * Departamento de Psiquiatría, Hospital \\ Universitario Kuopio, Kuopio \\ ** Departamento de Salud Pública y \\ Atención Primaria, Universidad de Kuopio, \\ Kuopio \\ *** Centro de Informática, Universidad de \\ Kuopio, Kuopio
}

FINLANDIA

\begin{abstract}
RESUMEN - Hemos investigado la prevalencia de una patología psiquiátrica común, y los factores asociados con ella en 91 pacientes con dolor osteoarticular de rodilla o de cadera, que han sido diagnosticados en consultas de Atención Primaria. El sesenta por ciento de los pacientes con osteoartritis sufrían una patología psiquiátrica común: estaban más deprimidos y mostraban síntomas de alexitimia con más frecuencia que el resto de los pacientes. Los análisis de regresión logística mostraron cómo el dolor que produce trastornos en el sueño nocturno, se asociaba de forma independiente con este tipo de patología psiquiátrica (odds-ratio 13,6,95\% intervalo de confianza 3,6-51). Creemos que debería examinarse la existencia de patologías psiquiátricas, en pacientes con osteoartritis de cadera o de rodilla y con dolor que altere el sueño nocturno.
\end{abstract}

\section{Introducción}

La osteoartritis de rodilla o de cadera provoca distintos niveles de discapacidad. Esta discapacidad física producida por la osteo- artritis, el dolor y la angustia psíquica están obviamente asociados. Mäkelä et al. (1993) mostraron cómo la osteoartritis de cadera y de rodilla estaban especialmente relacionadas con la necesidad, tanto de forma constante como ocasional, de asistencia. Por su 
parte Payne et al. (1994) no encontraron correlación entre los ingresos hospitalarios y la prevalencia de estas enfermedades. Y señalaron que la utilización de los servicios hospitalarios no puede considerarse como sustitutivo en el tratamiento de estas patologías.

Existen pocos estudios sobre pacientes con osteoartritis que sufran de patología psiquiátrica. Dexter y Brandt (1994) descubrieron que los síntomas depresivos en pacientes con osteoartritis no eran más importantes que los presentados por la población general, sin embargo, aquellos pacientes con osteoartritis que recibían tratamiento estaban más deprimidos que aquellos que no lo recibían. Vali y Walkup (1998) mostraron que los síntomas depresivos se manifestaban en los días en los que su actividad presentaba limitaciones debido a su osteoartritis. Pocos pacientes recibieron ayuda para solucionar sus problemas emocionales, descubriéndose en estos casos un menor cumplimiento del tratamiento.

Hay que partir de la base de que la mayoría de los pacientes con osteoartritis son tratados en consultas externas, y se sabe que, incluso las enfermedades mentales leves afectan profundamente el tratamiento de pacientes con enfermedades físicas (Wells et al. 1989). Pero se desconoce el alcance que pueda llegar a tener un trastorno psíquico en pacientes con osteoartritis. Sería útil poder identificar variables simples que ayudaran a los médicos de Atención Primaria a controlar esta patología psíquica en pacientes con osteoartritis, por lo tanto, el objetivo de este estudio es determinar la prevalencia de patología psiquiátrica en pacientes con osteoartritis de cadera o de rodilla en Atención Primaria, así como identificar las variables asociadas a ella.

\section{Material y método}

Intervinieron en este estudio en el año 1996 veintiocho médicos de familia pertenecientes a veinticinco Centros de Salud, que habían sido elegidos de forma aleatoria, en diferentes áreas en Finlandia. Los pacientes fueron tratados durante 4 semanas; una semana en cada estación del año. Se recogieron datos de todos los pacientes que acudieron a las consultas, y desde luego, datos específicos en el caso en que el dolor fuese la razón fundamental por la que se acudía a la consulta o bien si el paciente se quejaba de dolor de forma espontánea. El diseño del estudio ha sido descrito de forma detallada en otros artículos (Mäntyselkä et al., en prensa).

Explicaremos el procedimiento brevemente. Se entregó un cuestionario a todos los pacientes. Las preguntas estaban relacionadas con factores sociodemográficos y con la duración y frecuencia del dolor. Además se pidió a los pacientes que midiesen la intensidad del dolor que padecían en un momento determinado utilizando para ello una Escala Visual Análoga (rango de puntuación 0-100). También se preguntó a los pacientes sobre la medicación utilizada. El cuestionario incluía la Escala de Depresión de Beck de 13 items (rango de puntuación 0-39) (Beck et al. 1988) y la Escala de Alexitimia de Toronto (rango de puntuación 20-100) (Bagby et al. 1994, Honkalampi et al. 2000).

Cada uno de los médicos que tomó parte en el estudio elaboraba sus diagnósticos utilizando la clasificación ICD-10. La intensidad del dolor en cada paciente también era evaluada por cada médico utilizando la Escala visual análoga.

Se evaluó la prevalencia de manifestaciones psiquiátricas leves utilizando el Cuestionario de Salud General de Goldberg de 12 items, un instrumento breve pero válido 
para la detección de casos (Goldberg \& Williams 1991, Goldberg et al. 1997, Viinamäki et al. 2000), cuyas preguntas están relacionadas con la ansiedad, la depresión y la autoestima. Las respuestas se pueden valorar con puntuaciones totales de 0 a 12 . Una puntuación de 3 indica la probabilidad de manifestaciones psiquiátricas. Las puntuaciones inferiores indican que el paciente goza de una salud mental normal. Utilizamos este punto de corte para dividir a los pacientes en dos grupos, aquellos con salud mental normal y los que padecen de una patología psiquiátrica leve.

Durante el periodo de estudio se realizaron 6.047 visitas, pero hemos excluido los datos relacionados con 401 de estas visitas (400 pacientes no quisieron formar parte del estudio y en un caso faltaban datos). Del resto 2.237 visitas fueron a causa del dolor. y 91 pacientes fueron diagnosticados por su médico general de osteoartritis de cadera o rodilla (ICD-10, códigos M16 o M17).
El test "chi-cuadrado" se utilizó en análisis estadísticos de variables nominales y el test de "T de Student" en análisis de variables continuas. Se calcularon odds ratios con un $95 \%$ de intervalos de confianza, utilizando análisis de regresión logística multivariable con el fin de descubrir las asociaciones independientes de cada factor con esta patología psiquiátrica leve y para así controlar aquellos factores que pudieran dar lugar a confusión.

\section{Resultados}

En los 91 pacientes con osteoartritis estudiados, se descubrió que el $60 \%$ tenían manifestaciones psiquiátricas leves pero no se pudo asociar ninguna variable sociodemográfica o del estilo de vida con manifestaciones psiquiátricas leves. Tampoco se apreció ninguna relación entre la duración

Tabla I

Aspectos sociodemográficos y características clínicas de acuerdo con la presencia o ausencia de patología psiquiátrica leve en pacientes que sufren dolor osteoartrítico de cadera o de rodilla

\begin{tabular}{lccc} 
Variable & $\begin{array}{c}\text { Manifestaciones } \\
\text { psiquiátricas leves } \\
(\mathrm{n}=55)\end{array}$ & $\begin{array}{c}\text { Salud mental } \\
\text { normal } \\
(\mathrm{n}=36)\end{array}$ & Valor p \\
\hline Mujeres, \% & 71 & 72 & $\mathrm{~ns}$ \\
Edad, años (media \pm SD) & $66 \pm 11,2$ & $66 \pm 8,6$ & $\mathrm{~ns}$ \\
Casado, conviviente, \% & 64 & 64 & $\mathrm{~ns}$ \\
Jubilado, \% & 78 & 72 & $\mathrm{~ns}$ \\
Fumador, \% & 4 & 14 & $\mathrm{~ns}$ \\
No bebe alcohol, \% & 55 & 43 & $\mathrm{~ns}$ \\
Toma diariamente analgésicos, \% & 47 & 56 & $\mathrm{~ns}$ \\
Duración del dolor menos de 6 meses, \% & 57 & $50 \pm 20,3$ & $\mathrm{~ns}$ \\
Puntuación VAS (media \pm SD) & & $45 \pm 17,7$ & $\mathrm{~ns}$ \\
$\quad$ Opinión del paciente & $58 \pm 20,2$ & 48 & $<0,001$ \\
Opinión del médico & $49 \pm 17,4$ & $47 \pm 10,4$ & $<0,01$ \\
El dolor altera el sueño nocturno, \% & 91 & $2,6 \pm 2,2$ & \\
Puntuación TAS-20 (media \pm SD) & $53 \pm 13,3$ & & \\
Puntuación Beck-13 (media \pm SD) & $7,2 \pm 4,6$ & &
\end{tabular}


del dolor, el consumo regular de analgésicos o la intensidad del dolor (puntuación de la Escala Visual Análoga) y manifestaciones psiquiátricas leves. Los pacientes con enfermedades mentales leves estaban significativamente más deprimidos que otros pacientes y mostraban más síntomas de alexitimia. La mayoría de ellos manifestaron que el dolor les impedía dormir por la noche (tabla I). Ninguno de los pacientes tomaba medicación antidepresiva.

Utilizando un análisis de regresión logística para estudiar los factores asociados de forma independiente con manifestaciones psiquiátricas leves así como un modelo que incluía las variables de edad (años), sexo $($ masculino $=0$, femenino $=1)$, estado civil ( casado / conviviente $)=0$, otros $=1$ ), fuma ( sí $=0$, no $=1$ ) y alteración del sueño por la noche a causa del dolor $($ no $=0$, sí $=1$ ), se descubrió que la alteración del sueño por la noche a causa del dolor era la única que se asociaba de forma independiente con manifestaciones psiquiátricas leves (osteoartritis 13,6, 95\% CI 3,6-51). La escala de alexitimia de Toronto-20 y las puntuaciones obtenidas con la Escala de Depresión de Beck de 13 items no fueron incluidas en el modelo porque estaban fuertemente correlacionadas con las puntuaciones del Cuestionario de Salud General.

\section{Discusión}

Hemos encontrado que más de la mitad de los pacientes con osteoartritis de cadera o rodilla que acudieron a la consulta de un médico de familia padecían manifestaciones psiquiátricas leves, pero no hay datos disponibles de estudios relacionados con diseños similares para poder comparar. En la población general, la prevalencia de manifestacio- nes psiquiátricas leves variaba en el caso de los hombres finlandeses entre el 16 al $19 \%$ y en las mujeres entre el 21 al $25 \%$ durante 1994-1995 (Viinamäki et al. 2000). En la población general, el 2,4\% de las mujeres y el 2,3\% de los hombres estudiados, se habían planteado el suicidio durante los 12 meses anteriores y, de estos individuos, el $62 \%$ de las mujeres y el $61 \%$ de los hombres padecían manifestaciones psiquiátricas leves (Hintikka et al. 1998). Los pacientes con osteoartritis y dolor en la cadera o en la rodilla padecían, por tanto, patologías psiquiátricas leves con parecida frecuencia a la de los suicidas en la población general.

La validez de los diagnósticos clínicos utilizados en el estudio no pudieron ser confirmados. Esto supone una limitación. Sin embargo, los diagnósticos de osteoartritis de cadera o rodilla estaban basados en historias clínicas de Atención Primaria, hallazgos clínicos y métodos complementarios como radiografías. Por lo tanto, los diagnósticos de osteoartritis que se realizaron con nuestros medios fueron los que más probabilidades tenían de ser correctos, aunque puede que la enfermedad en sí no se haya llegado a diagnosticar en algunos pacientes.

La consistencia de nuestro estudio radica en el hecho de que el material utilizado es completo, ya que incluía a todos los pacientes que acudieron a consultas de Médicos de Familia durante el periodo de estudio a consecuencia del dolor. Por lo tanto, los resultados pueden considerarse fiables en lo que se refiere a pacientes con osteoartritis de cadera o rodilla que padecían dolor. Además la escala de alexitimia de Toronto-20 (Joukamaa et al. en prensa), la Escala de Depresión de Beck de 13 items (Beck et al. 1988, Viinamäki et al. 1995) y el Cuestionario de Salud General (GHQ-12) (Goldberg et al. 1997) son escalas de una validez y fiabilidad estudiadas ampliamente. 
Los resultados de nuestro estudio muestran que el empleo de analgésicos y la intensidad del dolor no tenían ninguna relación con patologías psiquiátricas leves. Además, la prevalencia de estas manifestaciones no estaba asociada con la duración del dolor. De esta forma, no se pueden utilizar estas variables para identificar a pacientes con osteoartritis que probablemente requerirán un examen psiquiátrico.

Los efectos adversos que produce el dolor en el sueño están relacionados fuerte e independientemente con manifestaciones psiquiátricas leves. La alteración del sueño es un síntoma característico de la depresión, sin embargo, la falta de sueño también puede predisponer a la depresión. Debido al diseño clínico cruzado de nuestro estudio, no es posible determinar si las manifestaciones psiquiátricas leves provocaban la pérdida de sueño o viceversa, en cambio, en pacientes con osteoartritis de cadera o de rodilla, el dolor que altera el sueño nocturno, indica claramente que la valoración del estado mental del paciente está justificada. Los médicos no deben pasar por alto la importancia de este síntoma común (Jordan et al. 2000).

Las manifestaciones psiquiátricas leves pueden pertenecer a diferentes síndromes psiquiátricos, de los cuales los más comunes son los Trastornos del Humor y de Ansiedad (Viinamaäki et al. 1995). Los Trastornos de Humor y otros síndromes psiquiátricos, normalmente requieren un tratamiento específico, pero la comorbilidad física puede afectar negativamente los resultados del tratamiento psiquiátrico (Keitnez et al. 1991, Wells et al. 1993) aunque no todos los estudios sostienen esta conclusión (Viinamäki et al. en prensa). Sin embargo, los pacientes con osteoartritis que sufren dolor asociado a manifestaciones psiquiátricas leves deben ser tratados con tanta eficacia como sea posible. Según nues- tros datos, ningún paciente tomaba medicación antidepresiva. En otras palabras, sus síntomas psiquiátricos no habían sido detectados y no estaban siendo tratados.

\section{Bibliografía}

BAGBY, R.M., PARKER, J.D.A., TAYLOR, G.J. The twenty-item Toronto Alexithymia Scale-1: item selection and cross-validation of the factor structure. J Psychosom Res 38: 23-32, 1994.

BECK,A.T.,STEER,R.A.,GARBIN, M.G. Psychometric properties of the Beck Depression Inventory: twenty years of evolution. Clin Psychol Rev 8: 77-100, 1988.

DEXTER, P.,BRANDT, K. Distribution and predictors of depressive symptoms in osteoarthritis. J Rheumatol 21: 279-286, 1994.

GOLDBERG, D.,WILLIAMS, P. A User's Guide to the General Health Questionnaire. Windsor:Basingstoke Press, 1991.

GOLDBERG, D.P., GATER, R., SARTORIUS, N., USTUN, T.B.,PICCINELLI,M.,GUREJE, D., RUTTER, C. The validity of two versions of the GHQ in the WHO study of mental illness in general health care. Psychol Med 27: 191-197, 1997.

HINTIKKA, J.,VIINAMÄKI, H., TANSKANEN, A., KONTULA, O., KOSKELA, K. Suicidal ideation and parasuicide in the Finnish general population. Acta Psy chiatr Scand 98: 23-27, 1998.

HONKALAMPI, K., HINTIKKA, J., TANSKANEN, A., LEHTONEN, J., VIINAMÄKI, H. Depression is strongly associated with alexithymia in the general population. J Psychosom Res 48: 99-104, 2000.

JORDAN, J.M., BERNARD, S.L., CALLAHAN, L.F., KINKADE, J.E., KONRAD, T.R., DEFRIESE, G.H.Selfreported arthritis-related disruptions in sleep and daily life and the use of medical, complementary, and self-care strategies for arthritis. Arch Fam Med 9: 143-149, 2000.

JOUKAMAA,M.,MIETTUNEN, J., KOKKONEN, P., KOSKINEN, M., JULKUNEN, J., KAUHANEN, J., JOKELAINEN, J.,VEIJOLA, J.,LÄKSY, K.,JÄRVELIN, M.R. Psychometric properties of the Finnish 20-item Toronto Alexithymia Scale. Nord J Psychiatry (in press).

KEITNER, G.I., RYAN, C.E., MILLER, J.W., KOHN, R., EPSTEIN, N.B. Twelve-month outcome of patients 
with major depression and comorbid psychiatric or medical illness (compound depression). Am J Psychiatry 148: 345-350, 1991.

MÄKELÄ, M, HELIÖVAARA, M., SIEVERS, K., KNEKT, P.,MAATELA, J.,AROMAA,A.Musculoskeletal disorders as determinants of disability in Finns aged 30 years or more. J Clin Epidemiol 46: 549-559, 1993.

MÄNTYSELKÄ, P., KUMPUSALO, E., AHONEN, R., KUMPUSALO, A., KAUHANEN, J., VIINAMÄKI, H., HALONEN, P., TAKALA, J. Pain as a reason to visit the doctor:a study in Finnish primary health care. Pain (in press).

PAYNE, J.N., COY, J., PATTERSON, S., MILNER, P.C. Is use of hospital services a proxy for morbidity? A small area comparison of the prevalence of arthritis, depression, dyspepsia,obesity and respiratory disease with inpatient admission rates for these disorders in England. J Epidemiol Commun H 48: 74-78, 1994.

VALI, F.M., WALKUP, J. Combined medical and psychological symptoms: impact on disability and health care utilization of patients with arthritis. Med Care 36: 10731084, 1998.

VIINAMÄKI, H., NISKANEN, L., KOSKELA, K. General Health Questionnaire and Beck Depression Scale as screening methods for psychiatric morbidity among the unemployed. Eur J Psychiatry 9: 209-215, 1995.
VIINAMÄKI, H., HINTIKKA, J., KONTULA, O., NISKANEN, L., KOSKELA, K. Mental health at population level during an economic recession in Finland. Nord $J$ Psychiatry 54: 177-182, 2000.

VIINAMÄKI, H., TANSKANEN, A., HINTIKKA, J., HASTAINEN, J., ANTIKAINEN, R., HONKALAMPI, K., HAATAINEN, K., KOIVUMAA-HONKANEN, H., SAARINEN, P.,LEHTONEN, J. Effect of somatic comorbidity on alleviation of depressive symptoms. Aust NZJ Psychiatry (in press).

WELLS, K.B., STEWART, A., HAYS, R.D., BURNAM,A., ROGERS, W., DANIELS, M.BERRY, S.,GREENFIELD, S., WARE, J. The functioning and well-being of depressed patients. Results from the medical outcomes study. J Am Med Assoc 262: 914-919, 1989.

WELLS, K.B., ROGERS, W., BURNAM,M.A.,CAMP, P. Course of depression in patients with hypertension, myocardial infarction,or insulin-dependent diabetes. Am J Psychiatry 150: 632-638, 1993.

Dirección para correspondencia:

Prof. Viinamäki Heimo

Departamento de Psiquiatría

Hospital Universitario Kuopio

Código postal 1777

FIN-70211 Kuopio

Correo electrónico: heimo.viinamaki@kuh.fi

FINLANDIA 